\title{
Production effects by form changes of autonomous decentralized FMSs with mind
}

\author{
Hidehiko Yamamoto \\ Mechanical Engineering, Gifu University, 1-1, Yanagido, Gifu Japan \\ Kakeru Yokoi \\ Human Information Systems Engineering, Gifu University, 1-1, Yanagido, Gifu Japan \\ Takayoshi Yamada \\ Mechanical Engineering, Gifu University, 1-1, Yanagido, Gifu Japan \\ E-mail: yam-h@gihu-u.ac.jp,yokoi1205@gmail.com,yamt@gihu-u.ac.jp
}

\begin{abstract}
In autonomous decentralized FMS, the number of agents and constant installation position has been studied in previous studies. Therefore, in this study, we are considering to change the position of the warehouse and the number of AGVs to redesign the factory form and evaluate the changes in the production of autonomous decentralized FMS. By being inspired by human mind, we are proposing Minimum Unit of Mind (MUM), a new method of controlling the behavior of AGVs to avoid the unexpected collision in autonomous decentralized FMS.
\end{abstract}

Keywords: Autonomous decentralized FMS, Mind, Route interference, AGV,

\section{Introduction}

We need to avoid collision among AGVs when we use autonomous decentralized FMS. There are some rules to avoid collisions, but these rules are not good enough to control the process of autonomous decentralized FMS because all situations can not be foreseen and rules are not described. Hence, the purpose of this study is to bring forward human mind to control the behavior of AGVs. In fact there are some limitations to control autonomous decentralized FMS in order to avoid the AGVs collisions, but in this study, we are trying to improve the FMS operations to keep a high efficiency.

To improve the efficiency and control the collision, we propose the development of behavioral control to avoid the collision by relying on the fact that simulating the human mind will help machines to make better decisions. In this study, we are considering to change the position of the product warehouse and change the number of AGVs to redesign the factory form and evaluate the changes on the production of autonomous decentralized FMS.

\section{Overview of autonomous decentralized FMS}

Fig.1 illustrates an autonomous decentralized FMS factory sketch. As Fig. 1 shows, the factory floor is divided into grid patterns, and AGVs are moving along these lines to carry parts to the warehouse and machine centers which is representing the system of autonomous factory to carry out the production. The autonomous decentralized FMS does not have a management mechanism to integrate the entire system, machining centers, AGV, product warehouse (PRW), parts warehouse (PAW). Each agent that configures the system autonomously determines the act by recognizing the purpose of the system by cooperating and negotiating to other agent. ${ }^{1}$ 


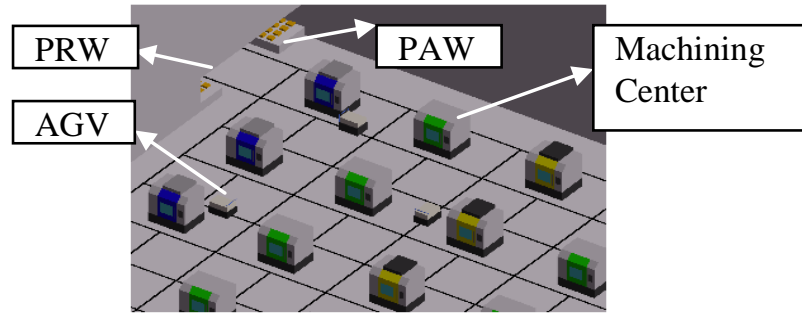

Fig. 1. Autonomous decentralized FMS

We describe the behavior of the AGV control of autonomous decentralized FMS factory. In autonomous decentralized FMS, each AGV determines the behavior autonomously. If any of the AGVs runs on the same line at the same time, they overlap and AGV path interference occurs. It is necessary to avoid such interference between AGVs and therefore, we have developed control rules for the system. If the number of AGVs increases, more unexpected events can occur because the rules become more complicated. Thus, it is impossible to generate rules that can be adapted to every situation in an autonomous decentralized FMS factory. This study focuses on the human mind, which can deal with unexpected events. We propose a mind model for the AGVs and develop a method to control their behavior by using a mind. The mind model considers two types of mind: an arrogant mind and a modest mind. AGV with arrogant mind takes the action approaching the destination forcibly. AGV with modest mind takes the action to give way to other AGV.

Fig.2 is a diagram about mind model. In this study, we call it Minimum Unit of Mind (MUM). Further, A1 and A2 is unit, $\mathrm{X}$ is load, and arrow is a stimulation vector. The threshold is determined by the unit.

If the internal value reaches the threshold, we call excited and if it does not reach, normal.

When a signal is sent to the unit, it sends a signal to the direction of the arrow when excited, and it is not sent when normal.

Load has the function to change the internal value of the unit. When the signal is sent to the load, the value of unit is decreased by the value of $\mathrm{X}$.

Stimulation vector is a line connecting the load and the unit. It gives a signal to the load or unit when the signal comes, when A1 keeps exited, we call modest mind, and when normal, we call arrogant mind.

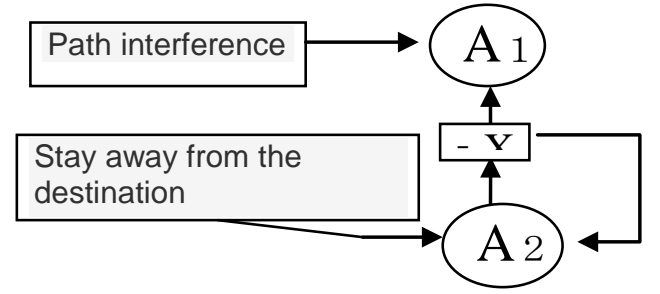

Fig. 2. Model of Mind

Next, we describe the internal functions of MUM. When the arrogant AGV has path interference, A1 is increased by 1 . Keeping the situation of the interference and being increased by 1 , the $\mathrm{A} 1$ value becomes the threshold value, and the AGV is changed to a modest one.

When AGVs with a modest mind keep giving a way, the value of $A 2$ is increased by 1 . When the situation is repeated an optional time and A2 becomes exited, a signal is sent to a load. The received load decreases the values of units $\mathrm{A} 1$ and $\mathrm{A} 2$ by optional values. Because of this, A1 and A2 are returned to normal and AGV with a modest mind is changed to one with an arrogant mind. In this way, when an arrogant mind is incorporated in the AGV, it is forceful in its motion. On the other hand, when a modest mind is incorporated in the AGV, it gives way to other AGVs.

\section{Impact on production conditions and FMS layout}

In this section we will investigate parameters such as capacity utilization, route interference and production outputs by applying following conditions:

\# Changing the position of the products warehouse

The product and part warehouses were immobilized in the previous studies and to prove that moveable warehouse method is effective, we decided to design five new factory layouts in this study.

\# Changing the number of machining centers and AGVs

In the previous studies, the number of AGV was allocated to five $\mathrm{AGVs}$ and the number of the machining centers was done with 24 units. We will change the AGV numbers from 2 to 9, and we adopt 24 units of machining center and 16 units in this study. 


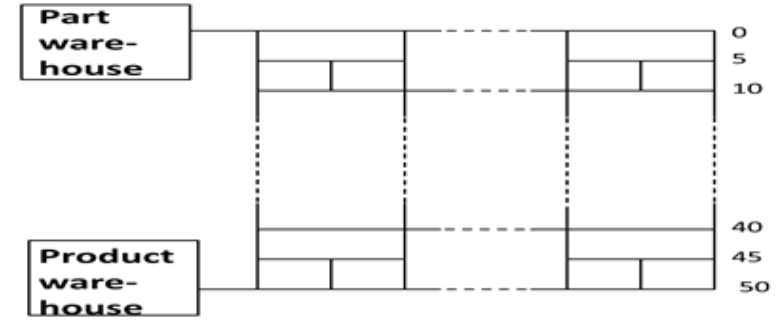

Fig. 3. Location of product warehouse $(0,50)$ layout

Fig. 3 is the layout of the production floor when the coordinates of the product warehouse are $(0,50)$.

\section{Simulation results and discussions}

Figure 4, Table 1 and Table 2 are the results of the simulation of production when the number of machining centers and AGVs are being changed. As the result shown, the more number of AGVs, the more productions will be produced. However, we have gotten a result that shows a little difference when the number of machining centers are 24 units and 16 units. In addition, in the first 30 minutes of the starting the simulation there is a high route interference number as shown in the Fig. 4. It is because, right after the starting the simulation, there is no part in the machine centers and all AGVs should go and pick the parts from the part warehouse simultaneously.

Table 1. Production outputs of MC number 16

\begin{tabular}{lllllllll}
\hline AGV & 2 & 3 & 4 & 5 & 6 & 7 & 8 & 9 \\
\hline data1 & 92 & 129 & 160 & 184 & 209 & 233 & 254 & 274 \\
data2 & 89 & 129 & 156 & 184 & 212 & 233 & 256 & 273 \\
data3 & 91 & 132 & 161 & 184 & 209 & 230 & 255 & 277 \\
\hline
\end{tabular}

Table 2. Production outputs of 24 MC number 24

\begin{tabular}{lllllllll}
\hline AGV & 2 & 3 & 4 & 5 & 6 & 7 & 8 & 9 \\
\hline data1 & 84 & 122 & 151 & 184 & 214 & 235 & 257 & 274 \\
data2 & 85 & 120 & 155 & 185 & 212 & 233 & 252 & 277 \\
data3 & 84 & 118 & 155 & 185 & 210 & 232 & 252 & 280
\end{tabular}

Next, we show the simulation results of autonomous decentralized FMS when the position of the product warehouse is changed to 5 different positions. Table 3 and 4 illustrate the utilization rate of MC when the number of AGVs are 8 and 5 and the number of MCs are set on 16.

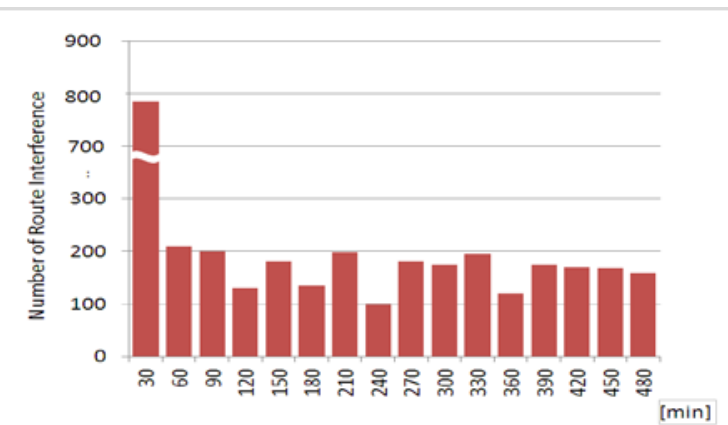

Fig. 4. Rout interference and its number of AGVs in every 30 minutes

Table 3. Utilization rate when the AGV number is 5

\begin{tabular}{ll}
\hline Product warehouse & Capacity utilization \\
\hline$(0,10)$ & 36.522 \\
$(0,25)$ & 37.187 \\
$(0,50)$ & 36.401 \\
$(60,0)$ & 36.15 \\
$(60,25)$ & 35.882 \\
$(60,50)$ & 35.248 \\
\hline
\end{tabular}

Table 4. Utilization rate when the AGV number is 8

\begin{tabular}{ll}
\hline Product warehouse & Capacity utilization \\
\hline$(0,10)$ & 49.864 \\
$(0,25)$ & 50.136 \\
$(0,50)$ & 50.306 \\
$(60,0)$ & 50.293 \\
$(60,25)$ & 50.075 \\
$(60,50)$ & 49.235 \\
\hline
\end{tabular}

When the AGVs number is 5 and the position of product warehouse is $(0,25)$ then the utilization rate of MC gives us a better result. Table 3 and 4 are shown the results of this simulation. However, when the number of AGVs is changed to 8 and the coordinates of PW are changed to $(0,50)$, the utilization rate gets a little bit better than the previous condition. On the other hand, when the AGVs number is 5 or 8 and PW coordinates are $(60,50)$, the utilization rate will be the lowest rate. Table 5 and 6 are shown the production outputs when the AGVs number is 5 and 8.

According to Table 5 and 6, PW with position (0, 50 ) has become the maximum production outputs when the AGVs number is 5 and 8 . In the case of 5 AGVs, the production outputs are increased. To compare with 8 AGVs, the ratio of increasing the products is not significant. The lowest result of production outputs which we have got in the simulation is the time when the PW position is $(60,50)$ with both 5 and 8 AGVs. 
Table 7 and 8 illustrate the route interference number in case of 5 and 8 AGVs.

Next, we show the simulation results when the position of the product warehouse is changed to 5 different positions. Table 3 and 4 illustrate the utilization rate of MC when the number of AGVs are 8 and 5 and the number of MCs are set on 16 .

Table 5. Production number of AGV number 5

\begin{tabular}{ll}
\hline Product warehouse & The production number \\
\hline$(0,10)$ & 182 \\
$(0,25)$ & 180 \\
$(0,50)$ & 191 \\
$(60,0)$ & 181 \\
$(60,25)$ & 180 \\
$(60,50)$ & 175 \\
\hline
\end{tabular}

Table 6. Production number of AGV number 8

\begin{tabular}{ll}
\hline Product warehouse & The production number \\
\hline$(0,10)$ & 250 \\
$(0,25)$ & 263 \\
$(0,50)$ & 263 \\
$(60,0)$ & 261 \\
$(60,25)$ & 253 \\
$(60,50)$ & 247 \\
\hline
\end{tabular}

Table 7. Route interference number of AGV number 5

\begin{tabular}{ll}
\hline Product warehouse & Route interference number \\
\hline$(0,10)$ & 997 \\
$(0,25)$ & 912 \\
$(0,50)$ & 729 \\
$(60,0)$ & 833 \\
$(60,25)$ & 850 \\
$(60,50)$ & 731 \\
\hline
\end{tabular}

Table 8. Route interference number of AGV number 8

\begin{tabular}{ll}
\hline Product warehouse & Route interference number \\
\hline$(0,10)$ & 2668 \\
$(0,25)$ & 2286 \\
$(0,50)$ & 2134 \\
$(60,0)$ & 2111 \\
$(60,25)$ & 2113 \\
$(60,50)$ & 1923 \\
\hline
\end{tabular}

According to Table 7 and 8 , the route interference number is the lowest result in case of 5 and $8 \mathrm{AGVs}$ and in the PW position $(60,50)$. As a result, the new production floors have a better result to compare with the previous production floors. Hence, autonomous distributed system that was newly created by this simulation, and the results we have got through this simulation show that the some layouts will be more efficient to use in the production floor.
There are different rules and techniques which have been studied by other researchers, the number of AGVs are limited so more number of AGVs cause more difficulties and collisions in the production floor in their study. In our research we have tried to improve the limitations and change the rules to be able to increase the number of AGVs as more as possible. To prove that our study is effective, we proposed MUM in this study.

In this section, we discuss the results of different layouts of the production floor, 5 and 8 AGVs, the number of parts, route interference number and MC utilization rate. According to mentioned Tables and Figures, the previous study with a PW position $(\mathrm{x}, \mathrm{y})=$ $(0,10)$ the route interference rate was high. In our study we change the layout of autonomous distributed system PW positions to $(0,25),(0,50),(60,0),(60,25),(60$, 50) and AGV route interference was reduced after applying changes. However the utilization rate of AGV is increased and interference number of $\mathrm{AGV}$ is reduced, but sometimes the production outputs will be lower. Sometimes due to a long distance between AGV and PW position, the AGV takes a lot of time to move between the two points, which is time consuming.

In our study, the result of simulation shows that production floor with $\mathrm{PW}$ position $(0,50)$ with 5 and 8 AGVs will have a better performance and will get a better result; we expect the production efficiency by following this pattern. There are several ways to design the layout and change the number of AGVs and MCs, however we have got a good result which seems efficient, but we suspect that there can be other ways to make optimum. It has also been found that it is necessary to perform the simulation, depending on the different conditions.

\section{Conclusions}

In conclusion, changing the number of MC and AGVs affected the production rate and capacity utilization. Applying the new conditions and layout designs has improved the production efficiency. Finding new optimal agent conditions by applying to autonomous decentralized FMS in order to improve the production efficiency should be done in a future work.

\section{Reference}

1.Hidehiko Yamamoto, Jaber Abu Qudeiri and M.A. Jamali, Real-time Control of Decentralized Autonomous Flexible Manufacturing Systems by using Memory and Oblivion, Lecture Notes of Computer Science , Springer, pp.252-259, ISBN 978-3-540-46535-5, Nov., 2006. 